\title{
Advances in Acoustic Sensing, Imaging, and Signal Processing
}

\author{
Jafar Saniie, ${ }^{1}$ Mario Kupnik, ${ }^{2}$ and Erdal Oruklu ${ }^{1}$ \\ ${ }^{1}$ Department of Electrical and Computer Engineering, Illinois Institute of Technology, Chicago, IL 60616, USA \\ ${ }^{2}$ Department of Electrical Engineering, Brandenburg University of Technology Cottbus, 03046 Cottbus, Germany
}

Correspondence should be addressed to Jafar Saniie, sansonic@ece.iit.edu

Received 5 July 2012; Accepted 5 July 2012

Copyright (c) 2012 Jafar Saniie et al. This is an open access article distributed under the Creative Commons Attribution License, which permits unrestricted use, distribution, and reproduction in any medium, provided the original work is properly cited.

The objective of this special issue is to address recent research trends and developments in the generation, acquisition, and processing of acoustic signals for industrial and medical applications. A substantial number of papers were submitted, and after a thorough peer review process, nine papers were selected to be included in this special issue. These papers cover important applications in acoustic sensing, including nondestructive testing of materials, enhanced ultrasonic imaging, underwater acoustic modeling, source mapping and communications. We believe that the original papers collected in this special issue highlight the contemporary topics in research related to acoustic sensing and imaging applications and will introduce readers to the latest advances in the field.

The paper by P. Etter presents a comprehensive review of contemporary underwater acoustic modeling methods. These advanced modeling techniques include forward and inverse applications, integrated-modeling approaches, nonintrusive measurements, and novel processing methods dealing with the changing ocean soundscape due to anthropogenic activity and natural factors.

The paper by P.-P. J. Beaujean and M. D. Staska, presents a computationally efficient underwater acoustic propagation model in a three dimensional rectangular duct closed at one end. The three-dimensional model presented in this paper provides a sufficient level of accuracy to be used in the simulation of an acoustic communication system operating between $15 \mathrm{kHz}$ and $33 \mathrm{kHz}$, with the benefit of low computing requirements.

The paper by E. Sarradj analyzes the beamforming steering vector formulations for three-dimensional acoustic source mapping. In particular, four different steering vector formulations from the literature are examined, and their theoretical backgrounds are discussed. It is shown that no formulation produces both correct acoustic source location and correct estimates of their strength. This paper identifies the two formulations that yield the correct estimation of the source location at the cost of an insignificant error in the estimated source strength.

The paper by J. Sandhu et al. reports on the application of acoustography using acoustooptical sensors for mapping ultrasonic fields radiated from ultrasonic transducers. This study is critical to assess the radiated ultrasonic field characteristics, which can be affected by many factors, such as piezoelectric material inhomogeneity, lack of bonding, electrode design and contact placement, acoustic lens quality, and wear plate uniformity and adhesion.

The paper by C. Quinsac et al., first introduces the theory of compressive sampling, which has been shown to reduce data below the Shannon-Nyquist limit. Next, the authors present different methods to perform compressive sampling in the context of ultrasound imaging with encouraging results in $2 \mathrm{D}$ and $3 \mathrm{D}$ ultrasound images.

The paper by Y. Zhang et al., discusses ultrasonic flaw imaging via multipath exploitation. In the proposed approach, by identifying multipaths that reflect at the known top and bottom surfaces, virtual sensors can be identified. The locations of these sensors permit visualizations of the shadowed regions, which otherwise are difficult to obtain from only direct reflection signals. The proposed multipath exploitation is supported by analysis and verified by experimental data.

The paper by J. S. Ullom et al. presents a new speckle reduction method for improving the contrast-to-noise ratio in ultrasonic B-mode images. The proposed method is based on resolution enhancement compression and a frequency 
compounding technique but it is enhanced to achieve higher axial resolution. Simulations and experimental measurements suggest that the proposed method can realize substantial improvements in terms of image visibility and enhance the boundaries between the target and the background.

The paper by Y. Lu et al. introduces a chirplet signal decomposition algorithm, based on fractional Fourier transform in order to analyze ultrasonic signals for NDE applications. Case studies and experimental results show that the proposed algorithm not only reconstructs the ultrasonic signal successfully, but also characterizes ultrasonic echoes and estimates echo parameters accurately.

The paper by R. Demirli et al., introduces a subspacebased approach for suppressing unwanted reverberations, enabling proper flaw detection and imaging. Two different cases are considered for the application of the proposed technique. The first case uses a set of flaw-free reference measurements of reverberation, whereas the second case is based on array measurements that contain flaws. For the first case, the clutter can be significantly removed with a negligible effect on the flaw echoes. In the second case that applies to ultrasound NDE imaging, it is demonstrated that the clutter can be mitigated by utilizing the array measurements without employing any reference data.

\section{Acknowledgment}

We would like to thank all the authors who contributed to this special issue. This publication would not be possible without the participation of our expert reviewers, who provided vital constructive feedback and criticism throughout the review process.

Jafar Saniie Mario Kupnik Erdal Oruklu 

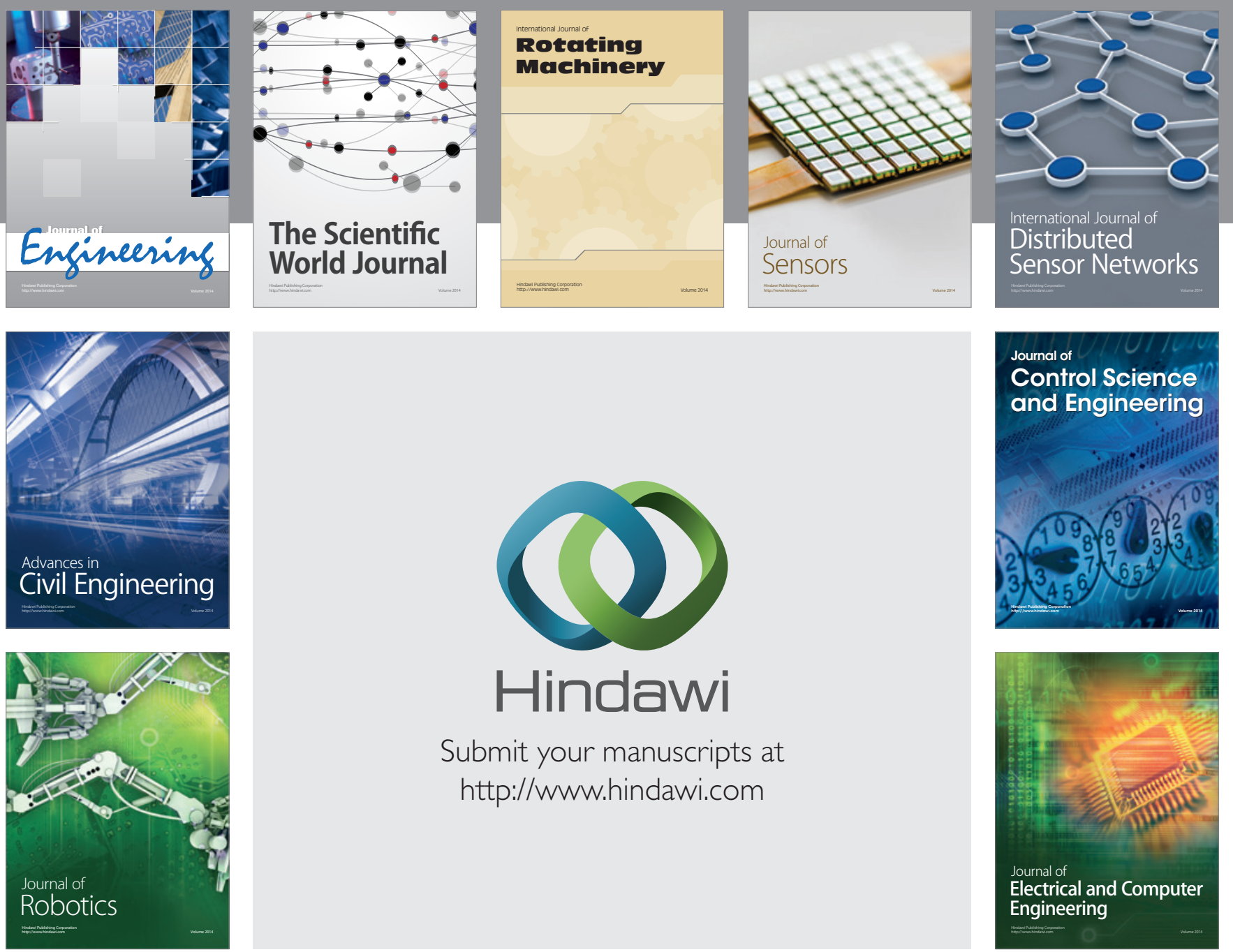

Submit your manuscripts at

http://www.hindawi.com
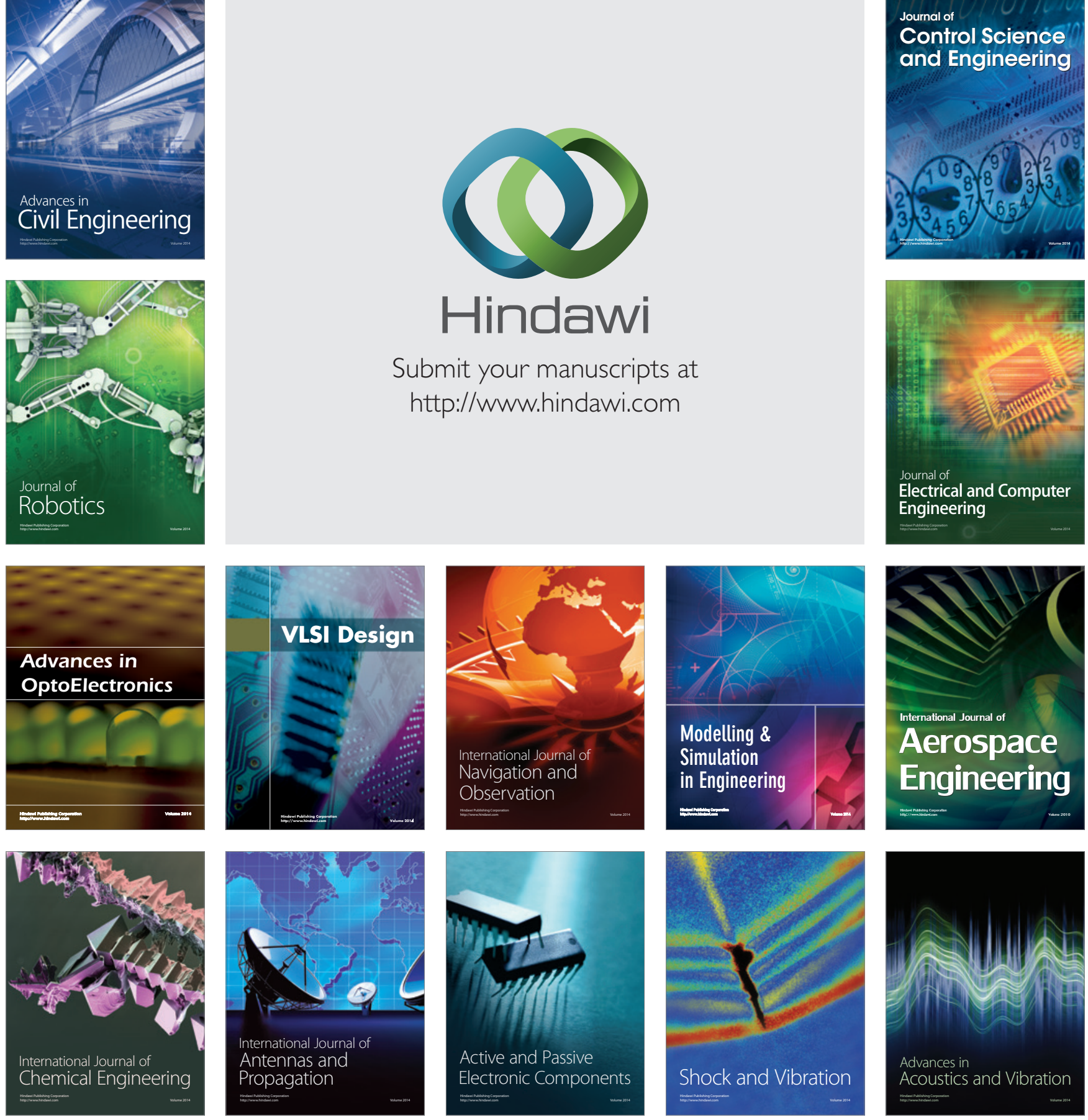